\title{
Blood Glutathione Peroxidase Activity in Relation with the Risk of Cardiovascular Diseases in Obese Women
}

\author{
Marie-Eve Lavoie ${ }^{1,2}$, Rémi Rabasa-Lhoret ${ }^{1,2,3}$, Sophie Ziai ${ }^{1,2}$ and Jean-Claude Lavoie ${ }^{1,4 *}$ \\ ${ }^{1}$ Department of Nutrition, Université de Montréal, Montreal, QC, Canada \\ ${ }^{2}$ Montreal Institute of Clinical Research (IRCM), Montreal, QC, Canada \\ ${ }^{3}$ Montreal Diabetes Research Center (MDRC) of Montreal University Hospital Research Center (CRCHUM), Montreal, QC, Canada \\ ${ }^{4}$ Research Center, CHU Sainte-Justine, Montreal, QC, Canada
}

\begin{abstract}
Objective: Oxidative stress plays a role in obesity-related diseases. We hypothesize that abnormalities of the glutathione system are associated with the initial phase leading to development of cardiometabolic abnormalities such as cardiovascular diseases and type 2 diabetes in apparently healthy obese women. By measuring different glutathione parameters, we expect to find a dichotomy that can discriminate between obese women who have such sub-clinical abnormalities.
\end{abstract}

Subjects: This is a cross-sectional analysis in 59 postmenopausal obese women. Total blood glutathione, glutathione peroxidase (GPx) and glutathione reductase activity, blood lipids, apolipoprotein B-100 (apoB), fasting and area under the curve (AUC) for glycemia and insulinemia during an oral glucose tolerance test, insulin sensitivity measurement and indices, serum inflammatory markers and carotid intima-media thickness (CIMT, by magnetic resonance imaging) were measured.

Results: Blood GPx activity had a bimodal distribution. Subjects were then divided into two groups according to their GPx activity (cut-off: $2.0 \mathrm{nmol} / \mathrm{min} / \mathrm{mg}$ protein, $P<0.01$ ). Age and BMI were similar between the groups. Women with higher GPx activity had $13 \%$ more apoB $(P=0.02), 10 \%$ higher glycemia AUC $(P=0.04)$, hepatic insulin resistance ( $28 \%$ and $25 \%$ higher HOMA and liver insulin resistance index values, $P<0.04)$ and increased CIMT by $8-13 \%(P=0.013)$ without evidence of inflammation, changes in blood lipids, and fasting glycemia and insulinemia.

Conclusion: Results suggest that a modification in the glutathione system is associated with insulin resistance and increased intima-media thickness, both of which are associated with cardiovascular disease risk. Blood GPx activity may be a parameter contributing in the identification of sub-clinical but clinically relevant asymptomatic cardiometabolic abnormalities in obese women.

Keywords: Glutathione peroxidase; Inflammation; Obesity; Carotid intima-media thickness; Insulin resistance

\section{Introduction}

Obesity is associated with an increase risk for cardiometabolic diseases such as atherosclerosis and type 2 diabetes [1]. The "common soil" hypothesis postulates that type 2 diabetes and cardiovascular diseases share common basis. Insulin resistance, for which oxidative stress is an early event and a potential underlying biochemical mechanism, is one of the most important aspects of this theory [2]. However, it is still unclear if oxidative stress is the cause or a consequence of these diseases. Furthermore, identifying obese subjects prone to develop cardiometabolic complications is challenging since a proportion of obese subjects appear to be at lower risk [3].

Oxidative stress results of an imbalance between the production and degradation of reactive oxygen species such as hydrogen peroxide $\left(\mathrm{H}_{2} \mathrm{O}_{2}\right)$. By modifying redox status of sensitive thiols, $\mathrm{H}_{2} \mathrm{O}_{2}$ modulates the biological function of several proteins including those involved in metabolic pathways [4]. Its intracellular concentration is regulated by its production mainly by the manganese superoxide dismutase (MnSOD) [5] and its reduction by glutathione peroxidase (GPx) in which reaction glutathione (GSH) is oxidized in a disulfide form (GSSG). A modification in GPx levels affects directly the intracellular level of peroxides; a slow-down of its activity allows higher intracellular concentration of peroxides whereas a stimulation of GPx activity leads to lower $\mathrm{H}_{2} \mathrm{O}_{2}$ concentration. On the other hand, in order to maintain the redox environment of the cell [6], the GSSG formed during the reduction of peroxides by GPx is recycled in GSH by glutathione reductase (GSSG-R) or is actively exported [7,8]. In a situation where the activity of GPx exceeds that of the GSSG-R, the GSSG is exported and the overall result is a loss in total glutathione level.

Because of its high reactivity with GPx and thiols [9], it is extremely difficult to detect, in vivo, a physiological modification in $\mathrm{H}_{2} \mathrm{O}$ concentration. However, the activity of GPx and GSSG-R as well as the level of total glutathione are accessible especially in erythrocytes, cells without nuclear capacity to restore homeostasis. Therefore, a modification in the blood glutathione system can be an early biomarker of chronic oxidative stress that can be an early step in the development of cardiometabolic complications.

The objectives of the study were to establish frequency distribution of glutathione and glutathione enzymes, and to compare a large panel of clinical, biochemical cardiometabolic risk factors and early

*Corresponding author: Jean-Claude Lavoie, Department of Nutrition, Universite de Montréal, Research Center, CHU Sainte-Justine, 3175 Côte Ste-Catherine, Montreal, QC, Canada, H3T 1C5, Tel: (514) 345-4931 \#3940; Fax: (514) 345-4801; E-mail: jean-claude.lavoie@umontreal.ca

Received July 25, 2011; Accepted August 18, 2011; Published September 08 2011

Citation: Lavoie M, Rabasa-Lhoret R, Ziai S, Lavoie J (2011) Blood Glutathione Peroxidase Activity in Relation with the Risk of Cardiovascular Diseases in Obese Women. J Diabetes Metab 2:136. doi:10.4172/2155-6156.1000136

Copyright: ( 2011 Lavoie M, et al. This is an open-access article distributed unde the terms of the Creative Commons Attribution License, which permits unrestricted use, distribution, and reproduction in any medium, provided the original author and source are credited. 
markers of atherosclerosis between obese subjects classified according to their variation in blood level of glutathione and/or activity of GPx and GSSG-R. A novelty of the study resides in the notion that a disturbed oxidative/reductive state could play a role in the initiation of cardiometabolic diseases such as atherosclerosis and type 2 diabetes. We hypothesized that, in a risky population such as obese subjects, a variance in the glutathione system can serve as an early marker of metabolic perturbations leading to cardiometabolic diseases.

\section{Methods}

\section{Subjects}

The study subjects examined were enrolled between 2006 and 2007 in the Complications Associated with Obesity (CAO) Study ( $n=59)$. The goal of the $\mathrm{CAO}$ project is to investigate different factors involved in the occurrence of obesity associated cardiometabolic complications. This study was conformed to the principles outlined in the Declaration of Helsinki as revised in 2000 and was approved by the Université de Montréal Ethics Committee. All subjects gave written informed consent. The baseline data of all subjects were used in the present analysis.

The inclusion/exclusion criteria of the CAO study were reported elsewhere [10]. Briefly, post-menopausal women were included in the study if they 1) were obese with BMI between 30 to $40 \mathrm{~kg} / \mathrm{m}^{2}$, 2) aged between 55 to 70 years old, 3) had biological confirmation of the menopause status without taking hormone replacement therapy, 4) were non diabetic and 5) non smokers, and 6) were not taking medications known to interfere with metabolism except stable hypothyroidism replacement therapy. All participants were free of chronic or inflammatory diseases and no history of alcohol or drug abuse.

\section{Body composition}

Standing height was measured to the nearest $0.1 \mathrm{~cm}$ with a wall stadiometer (Perspective Enterprises, Portage, MI) following standard techniques. Body weight and composition were measured by dualenergy X-ray absorptiometry with a LUNAR Prodigy system (software version 6.10.019; General Electric Lunar Corporation, Madison, WI). Measurements were conducted in the morning while the subjects were in a fasted condition. BMI was calculated as body weight $(\mathrm{kg}) / \mathrm{height}$ $(\mathrm{m})^{2}[11]$.

\section{Blood analysis}

Venous blood samples were collected at baseline of a $75 \mathrm{~g}$ oral glucose tolerance test (OGTT) and every 30 min for 2 hours. Glucose and fasting plasma cholesterol, HDL-cholesterol and triglycerides were analyzed on the Cobas Integra 400 (Roche Diagnostic, Montreal, QC, Canada). LDL-cholesterol was calculated according to the Friedewald equation [12]. Insulin levels were determined in duplicate by radioimmunoassay specific for human insulin (Linco, St-Charles, MO, USA). Apolipoprotein B-100 (apoB) was assessed by immunonephelometry on an Image Analyzer (Beckman-Coulter, Villepinte, France).

Serum high sensitivity C-reactive protein (hsCRP), orosomucoid and haptoglobin were assessed by immunonephelometry on an Image Analyzer (Beckman-Coulter, Villepinte, France). Eleven women with hsCRP $>10 \mathrm{mg} / \mathrm{L}$ were excluded from the analysis in this study because such an elevated hsCRP suggests an acute inflammatory response [13]. Serum IL-6 was measured in duplicates using a commercial highly sensitive ELISA kit (Quantikine, Minneapolis, MN, USA).

\section{Insulin sensitivity}

Insulin sensitivity was evaluated by three indices calculated from the OGTT values: 1) the fasting HOMA according to the formula of Matthews et al. [14], 2) the liver insulin resistance index according to the formula of Abdul-Ghani et al. [15] and 3) the SI is $_{\text {OGTT index }}$ mainly exploring muscle insulin sensitivity according to the formula of Bastard et al. [16]. Insulin sensitivity was also assessed by measuring the glucose disposal rate from the hyperinsulinemic euglycemic clamp as previously described [17]

\section{Glutathione systems}

Total glutathione was measured in whole blood by using a recycling enzymatic assay as previously described $[18,19]$. Glutathione peroxidase [20] and glutathione reductase [19,21] activities were determined by spectrophotometric methods.

\section{Carotid intima-media thickness}

Magnetic resonance imaging (MRI) was performed on a 1.5-T whole-body MRI system (Signa CV/i; GE Medical Systems; $40 \mathrm{mT} / \mathrm{m}$; SR150). A 4-element (2 elements on the right side of the neck and 2 elements on the left side) phased-array coil was used for carotid imaging [22]. After localization with a fast-gradient-echo sequence, all images were obtained with a double-inversion recovery (i.e. blackblood), fast spin-echo sequence with ECG-gating during free breathing. A total of 25 to 30 transverse images centered at the carotid bifurcation were taken. Imaging parameters were as follows: repetition time, 2 RR intervals; echo time, $12 / 45 \mathrm{~ms}$ (proton density-weighted/T2-weighted); field of view, $12 \mathrm{~cm}$; slice thickness, $3 \mathrm{~mm}$; no interslice gap; acquisition matrix, 256x256; no phase wrap; number of signals averaged, 1/2 (proton density-weighted/T2-weighted); echo train-length, 32; receiver bandwidth, $\pm 64 \mathrm{kHz} ; 512$ zero filling. A chemical shift suppression pulse was used to suppress the signal from perivascular fat.

\section{Statistical analysis}

Frequency distribution was drawn for total blood glutathione and specific activity of GSSG-R and GPx. Anthropometric and blood parameters were compared by using Student $t$-test. For hsCRP, the eleven women with values $>10 \mathrm{mg} / \mathrm{L}$ were excluded from the analysis of this marker, remaining 25 and 23 subjects in the Low and High GPx activity groups respectively. The vessel wall thickness was compared by using an ANOVA. A difference with an alpha error $(P)<0.05$ was considered as significant. In absence of significant differences, a statistical power (1-beta) $>80 \%$ enabled us to affirm that this parameter does not vary between the two groups. All data are presented as the mean \pm SEM.

\section{Results}

The first objective was to verify the frequency distribution of glutathione or glutathione enzymes. Figure 1 shows that only GPx activity had a bimodal distribution. Therefore, the subjects were separated in two groups according to the cut-off of $2.0 \mathrm{nmol} / \mathrm{min} /$ mg of protein: low $(1.43 \pm 0.04)$ and high GPx activity $(2.59 \pm 0.07)$ $(P<0.001)$. Total glutathione in blood was lower in the high GPx activity group by $20 \%(P<0.001)$ (Table 1$)$, suggesting a greater consumption of peroxides. No difference was found in GSSG-R activity between groups but the statistical power was low $(1-\beta=19 \%)$.

Table 2 indicates that age, body weight, BMI, systolic and diastolic blood pressure and serum inflammatory markers were comparable ( $1-\beta \geq 89 \%)$ between groups. No differences were identified for fasting 
A.

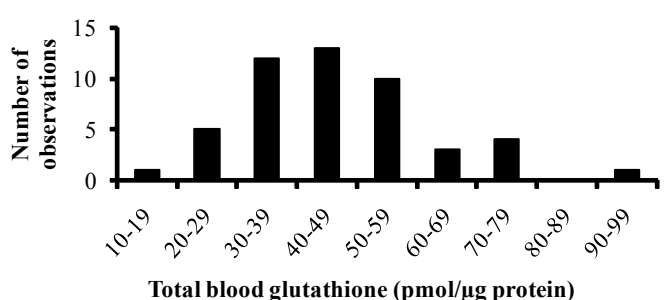

B.

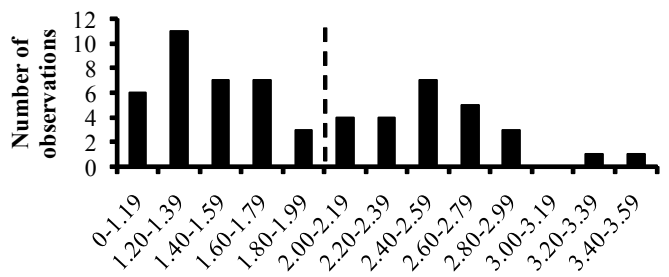

Blood glutathione peroxidase (nmol/min/mg protein)

C.

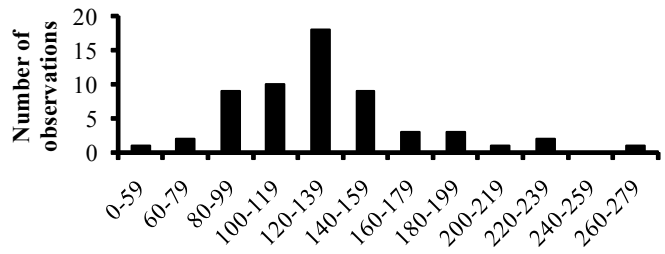

Blood glutathione reductase ( $\mathrm{pmol} / \mathrm{min} / \mathrm{mg}$ protein)

Figure 1: Frequency distribution of total glutathione (A), glutathione peroxidase activity (B) and glutathione reductase activity (C) in blood from the entire cohort. Values are presented as mean \pm SEM. Only glutathione peroxidase activity presented a bimodal distribution. The dash line represents the cut-off used to separate low GPx from high GPx.

A. Low GPx activity -High GPx activity

$$
\text { }
$$

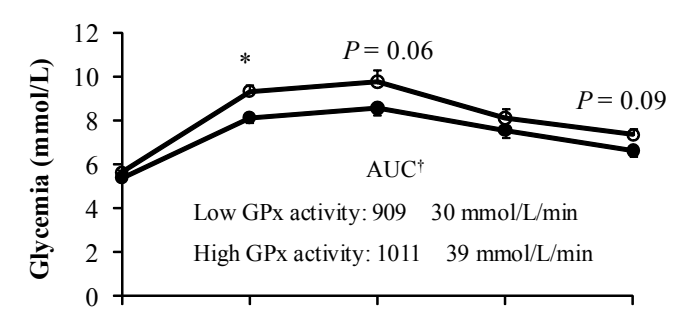

B.

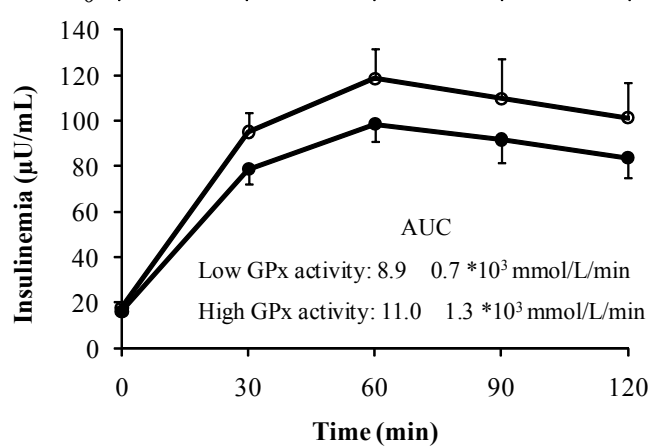

Figure 2: Glycemia (A) and insulinemia (B) during an OGTT in Low and High GPx activity subjects. Values are presented as mean \pm SEM. *Significantly different between groups $(P=0.04)$. ${ }^{\dagger}$ Significantly different between groups. AUC: Area under the curve.

and 2-hours glucose after the OGTT but the power was low $(1-\beta \leq 20 \%)$ (Table 2 and Figure 2). In contrast, area under the curve (AUC) for glycemia during the OGTT was greater in high GPx by $10 \%(P=0.04)$ (Figure 2).

Insulin resistance can affect different tissues in various ways that can be evaluated by various methods [23] (Table 2). No differences were observed for glucose disposal measured during the hyperinsulinemic-euglycemic clamp $(1-\beta=84 \%)$, fasting insulin $(1-\beta$ $=85 \%$ ) and peripheral mainly muscle insulin sensitivity as assessed by the $\mathrm{SI}_{\text {is }}$ OGTT index with however a low statistical power $(1-\beta \leq 66 \%)$. Despite no significant difference in fasting plasma glucose and insulin, subjects in the high GPx group presented a 28\% higher HOMA value $(P=0.04)$ and $25 \%$ higher liver insulin resistance index value $(P=0.03)$.

Triglycerides and HDL-cholesterol $(1-\beta \geq 92 \%)$ as well as LDLcholesterol $(1-\beta \leq 44 \%)$ values were comparable in both groups (Table 2 ). However, apoB was more abundant by $13 \%$ in high GPx group $(P<0.02)$.

Figure 3 shows that the thickness of the intima-media for both left and right carotid arteries was significantly higher $(P<0.05)$ in the high GPx group (right vessel wall $=0.28 \pm 0.01 \mathrm{vs} 0.24 \pm 0.01 \mathrm{~cm}^{2}$, left vessel wall $=0.26 \pm 0.01$ vs $\left.0.24 \pm 0.01 \mathrm{~cm}^{2}\right)$.

\section{Discussion}

In this cohort of subjects at high risk of cardiometabolic complications, based on their BMI and age (obese postmenopausal women) but without established cardiometabolic complications, we examined if a variance in the glutathione system could be an early biomarker for cardiometabolic abnormalities. Our results show that high GPx activity is associated with numerous potentially clinically relevant cardiometabolic abnormalities.

The fact that total glutathione is lower in higher GPx group suggests that GPx is active to metabolize peroxides $\left(\mathrm{H}_{2} \mathrm{O}_{2}\right)$ and hence the glutathione is consumed. In this group, the higher ratio of GPx to GSSG-R supports the fact that generation of GSSG following the GPx activity was higher than the recycling activity of GSSG-R. Considering that an excess of GSSG is toxic to the cells, GSSG in excess is actively exported in order to maintain the redox potential of the cells; explaining the low glutathione level observed in the high GPx group. Our results are in line with those of Surapaneni \& Venkataramana who reported elevated GPx activity and lower concentrations of total glutathione along with increased lipid peroxidation, an indication of increased production of reactive oxygen species, in osteoarthritis patients [24]. As suggested by the authors, the increased activity of the antioxidant enzyme GPx "may be a compensatory regulation in response to increased oxidative stress" [24].

The women in the high GPx group had also a greater insulin

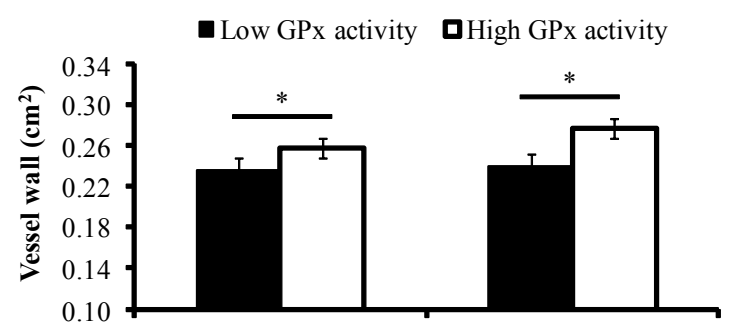

Figure 3: Carotid intima-media thickness from left and right artery in Low and High GPx activity subjects. Values are presented as mean \pm SEM. *Significantly different between groups for both left and right carotids $(P=0.013)$. 
Citation: Lavoie M, Rabasa-Lhoret R, Ziai S, Lavoie J (2011) Blood Glutathione Peroxidase Activity in Relation with the Risk of Cardiovascular Diseases in Obese Women. J Diabetes Metab 2:136. doi:10.4172/2155-6156.1000136

Page 4 of 6

\begin{tabular}{|c|c|c|c|c|c|}
\hline Characteristics & Low GPx activity ( $n=34)$ & High GPx activity (n=25) & $P$ value & Beta value & 1-Beta (\%) \\
\hline 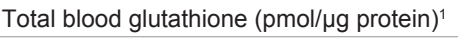 & $50 \pm 3$ & $40 \pm 2$ & $<0.001$ & & \\
\hline GPx (nmol/min/mg protein) & $1.43 \pm 0.04$ & $2.59 \pm 0.07$ & $<0.001$ & & \\
\hline GSSG-R (pmol/min/mg protein) & $124 \pm 5$ & $141 \pm 10$ & 0.11 & 0.813 & 19 \\
\hline GPX/GSSG-R & $12 \pm 1$ & $21 \pm 2$ & $<0.001$ & & \\
\hline
\end{tabular}

Mean \pm SEM

GPx: glutathione peroxidase (specific activity), GSSG-R: glutathione reductase (specific activity ${ }^{1}$ whole cohort: $n=49$, Low GPx activity: $n=30$, High GPx activity: $n=19$

Table 1: Glutathione metabolism of the subjects with either low or high GPx activity.

\begin{tabular}{|c|c|c|c|c|c|}
\hline Characteristics & Low GPx activity ( $n=34)$ & High GPx activity $(n=25)$ & $P$ value & Beta value & 1-Beta (\%) \\
\hline Age (years) & $56 \pm 1$ & $56 \pm 1$ & 0.92 & 0.03 & 97 \\
\hline Body weight (kg) & $91 \pm 2$ & $91 \pm 3$ & 1.00 & 0.03 & 97 \\
\hline BMI $\left(\mathrm{kg} / \mathrm{m}^{2}\right)$ & $34.5 \pm 0.5$ & $34.6 \pm 0.6$ & 0.85 & 0.03 & 97 \\
\hline Systolic BP (mm Hg) & $125 \pm 3$ & $126 \pm 3$ & 0.73 & 0.04 & 96 \\
\hline Diastolic BP (mm Hg) & $79 \pm 3$ & $79 \pm 1$ & 0.96 & 0.03 & 97 \\
\hline \multicolumn{6}{|l|}{ Blood lipids and apolipoproteins } \\
\hline Cholesterol (mmol/L) & $5.21 \pm 0.15$ & $5.57 \pm 0.18$ & 0.13 & 0.66 & 34 \\
\hline LDL-C (mmol/L) ${ }^{1}$ & $3.09 \pm 0.14$ & $3.41 \pm 0.17$ & 0.15 & 0.56 & 44 \\
\hline HDL-C (mmol/L) & $1.52 \pm 0.05$ & $1.48 \pm 0.06$ & 0.67 & 0.08 & 92 \\
\hline Triglycerides $(\mathrm{mmol} / \mathrm{L})^{1}$ & $1.30 \pm 0.09$ & $1.32 \pm 0.09$ & 0.33 & 0.04 & 96 \\
\hline apoB (g/L) & $0.89 \pm 0.04$ & $1.02 \pm 0.03$ & 0.02 & & \\
\hline \multicolumn{6}{|l|}{ Glucose status } \\
\hline Fasting glycemia (mmol/L) & $5.4 \pm 0.1$ & $5.7 \pm 0.1$ & 0.13 & 0.80 & 20 \\
\hline Fasting insulinemia $(\mu \mathrm{U} / \mathrm{mL})^{2}$ & $16.1 \pm 1.2$ & $17.7 \pm 1.6$ & 0.41 & 0.15 & 85 \\
\hline HOMA-IR & $3.48 \pm 0.29$ & $4.83 \pm 0.60$ & 0.04 & & \\
\hline Liver insulin resistance index $\left(x 10^{4}\right)$ & $29.3 \pm 2.4$ & $39.0 \pm 4.0$ & 0.03 & & \\
\hline $\mathrm{SI}_{\text {is }} \mathrm{OGTT}$ & $0.263 \pm 0.003$ & $0.257 \pm 0.004$ & 0.18 & 0.34 & 66 \\
\hline Glucose disposal (mg/min/kg) & $12.5 \pm 0.7$ & $11.6 \pm 0.9$ & 0.38 & 0.16 & 84 \\
\hline \multicolumn{6}{|l|}{ Serum inflammatory markers } \\
\hline IL-6 (pg/mL) & $1.7 \pm 0.2$ & $1.6 \pm 0.7$ & 0.65 & 0.04 & 94 \\
\hline $\mathrm{hsCRP}(\mathrm{mg} / \mathrm{L})^{3}$ & $4.4 \pm 0.5$ & $4.4 \pm 0.6$ & 0.94 & 0.03 & 97 \\
\hline Orosomucoid (g/L) & $0.87 \pm 0.02$ & $0.87 \pm 0.04$ & 0.93 & 0.03 & 97 \\
\hline Haptoglobin (g/L) & $1.6 \pm 0.1$ & $1.5 \pm 0.1$ & 0.46 & 0.11 & 89 \\
\hline
\end{tabular}

Mean \pm SEM

BMI: body mass index, BP: blood pressure, LDL-C: low density lipoprotein cholesterol, HDL-C: high density lipoprotein cholesterol, apoB: apolipoprotein B, glucose disposa rate calculated from the hyperinsulinemic euglycemic clamp, hsCRP: high sensitivity C-reactive protein

${ }^{1}$ whole cohort: $n=58$, Low GPx activity: $n=34$, High GPx activity: $n=24$

2whole cohort: $n=57$, Low GPx activity: $n=32$, High GPx activity: $n=25$

${ }^{3}$ whole cohort: $n=48$, Low GPx activity: $n=25$, High GPx activity: $n=23$

Table 2: Anthropometric, blood pressure, blood lipid profile, glucose status and inflammatory markers of subjects with either low or high GPx activity

resistance, as shown by the HOMA value. Similar results have been reported in severely obese individuals. Those with higher insulin resistance, as estimated by the HOMA, had a greater GPx activity compared to those with a lower insulin resistance [25]. In patients with chronic hepatitis C, Oliviera et al. [26] reported that those with higher insulin resistance, also assessed by the HOMA index, had a greater GPx activity and a lower total blood glutathione concentration compared to those with lower insulin resistance. Furthermore, in our study, the insulin resistance predominantly affected the liver as shown by the HOMA (a fasting index primarily exploring liver insulin resistance) as well as by a liver insulin resistance index (Table 2). This translated into a higher glycemia AUC during an OGTT, probably because of lower hepatic glucose suppression, a well-known early consequence of hepatic insulin resistance [27-29]. In animal models of insulin resistance induced by diet, liver is the first organ to show resistance to insulin action, followed by the adipose tissue and the muscles [2729]. Despite the fact that the increased values in HOMA and glycemia AUC during an OGTT in the high GPx group might seem modest, considering that, by design, all the subjects were obese but without established cardiometabolic complications, these modifications appear to be early in the development of cardiometabolic diseases such as cardiovascular diseases and type 2 diabetes. In the general population, the insulin resistance estimated by the HOMA index is a predictor of future symptomatic cardiovascular diseases independently of the classic and several nontraditional risk factors [30]. Taken all together, these results indicate that women with greater GPx activity present an altered glucose tolerance and insulin sensitivity that, in addition to their obesity status, contribute to their increased risk of cardiometabolic diseases.

The increased apoB100-related lipoprotein level in the high GPx group, which reflects the number of LDL particles, is also in line with hepatic insulin resistance [31] and is a well established causal factor in the development of atherosclerosis [32]. Indeed these patients demonstrate significant structural atherosclerosis damage demonstrated by the increased thickness of the blood vessels assessed by MRI (Figure 3). All these cardiometabolic risk factors and early atherosclerosis abnormalities are consistent with the reported positive association between high levels of blood GPx activity and cardiovascular events in humans [33]. The absence of difference in blood lipid 
profile between the two groups may certainly be a consequence of the inclusion criteria of the study that recruited only women who did not take any medication, thus excluding those with an altered blood lipid profile. Nevertheless, our results suggested that even in absence of an impaired classic blood lipid profile, a greater GPx activity may help to identify women with an increased risk of future cardiovascular diseases as evidenced by greater apoB concentrations.

The absence of inflammation, which is induced by oxidative stress [34], may seem counter-intuitive when considering that greater GPx activity might be a compensatory response to the enhance peroxide production, which supports the idea of oxidative stress. However, the greater activity of the antioxidant enzyme GPx may rather result in a "reductive" stress by compensating the higher production of peroxides. While the oxidative stress is the imbalance between the production and the degradation of the reactive oxygen species in favor of an overproduction, a "reductive" stress is the result of a greater antioxidant response (GPx activity) to the production of peroxides, thus in favor of higher rate of degradation. This concept has recently been supported by a study of Zhang et al., in which they found that a "reductive" stress may play an important role in the development of cardiomyopathy [35]. They induced a reductive stress in mice by overexpressing an antioxidant protein, Hsp27, and found that these mice development a cardiomyopathy. The levels of reactive oxygen species in the heart of these mice were reduced and the GPx tissue expression and activity were increased [35]. Furthermore, in mice, feeding a high-fat diet, Matsuzawa-Nagata et al. [36] found that the increased reactive oxygen species production induced by the diet appears before increases in TNF-alpha and free fatty acid concentrations. Moreover, this increase of reactive oxygen species was found in liver but not in adipose tissue, which could be a result of the increase in GPx expression and activity in that tissue [36]. It is known that increased production of reactive oxygen species leads to insulin resistance [37]. Our results support these findings as we found evidence of insulin resistance in the liver only. Taken all together, the liver appears to be a central organ and hepatic dysfunction results in gluco-lipidic abnormalities in which increased postprandial glucose excursion and high apoB might be early events already associated with structural atherosclerosis changes. The progression of the metabolic changes will lead to a dysregulation of lipid metabolism, oxidative stress and inflammation, the well-known sequence of events. Considering the transversal design of the study, our results suggest that a "reductive" stress may be one of, if not the first, event starting the metabolic changes leading to cardiometabolic complications. Further studies are needed to detail this sequence. The association between blood GPx activity and liver and vascular changes involved in cardiometabolic complications suggests that modification in activity of GPx is systemic. Because the promoter region of GPx gene has sites for methylation [38,39], an epigenetic modification of this gene remains an attractive hypothesis.

Our findings derived from a homogenous sample of wellcharacterized post-menopausal women who were, despite their numerous risk factors, free of any known inflammatory or chronic disease. However, we must be careful before extrapolating our results to all obese individuals. Nevertheless, our results suggest that a reductive stress, which is defined here as a lower intracellular concentration of $\mathrm{H}_{2} \mathrm{O}_{2}$ than normal homeostasis, can be an early event inducing modifications in metabolism. Thus, measurement of blood GPx activity may identify obese but otherwise healthy individuals with higher cardiometabolic risk and help to detect the progression of pathologies associated with insulin resistance. This notion should be reinforced by a prospective a study.

\section{Acknowledgements}

We acknowledge the help of A. Tardif, Dr. J-P Bastard and Dr. S Fellah with their assistance in measuring the inflammatory markers for which BeckmanCoulter, France kindly donated antibodies and analysis, J. Levasseur (RN) for the coordination of the study and Dr. J-C Tardif's laboratory for the measurement of the endothelial function and the carotid artery intima-media thickness. This study was supported by grants from Genome Canada-Quebec [CAO, Complications Associated with Obesity Project from the DGDG grant: Diabetes genes Discovery Group to R.R.L.]; the Fonds de la Recherche en Santé du Québec [scholarship to M.E.L. and S.Z.] and the Canadian Institutes of Health Research [scholarship to S.Z.].

\section{Conflict of Interest}

The authors declare no conflict of interest.

\section{References}

1. Zalesin KC, Franklin BA, Miller WM, Peterson ED, McCullough PA (2008) Impact of obesity on cardiovascular disease. Endocrinol Metab Clin North Am 37: 663-684, ix.

2. Stern MP (1995) Diabetes and cardiovascular disease. The "common soil" hypothesis. Diabetes 44: 369-374

3. Primeau V, Coderre L, Karelis AD, Brochu M, Lavoie ME, et al. (2011) Characterizing the profile of obese patients who are metabolically healthy. Int $\mathrm{J}$ Obes (Lond) 35: 971-981.

4. Paulsen CE, Carroll KS (2010) Orchestrating redox signaling networks through regulatory cysteine switches. ACS Chem Biol 5: 47-62.

5. Buettner GR, Ng CF, Wang M, Rodgers VG, Schafer FQ (2006) A new paradigm: manganese superoxide dismutase influences the production of $\mathrm{H} 2 \mathrm{O} 2$ in cells and thereby their biological state. Free Radic Biol Med 41: 1338 1350.

6. Schafer FQ, Buettner GR (2001) Redox environment of the cell as viewed through the redox state of the glutathione disulfide/glutathione couple. Free Radic Biol Med 30: 1191-1212.

7. Lauterburg BH, Adams JD, Mitchell JR (1984) Hepatic glutathione homeostasis in the rat: efflux accounts for glutathione turnover. Hepatology 4: 586-590.

8. Srivastava SK, Beutler E (1969) The transport of oxidized glutathione from the erythrocytes of various species in the presence of chromate. Biochem $\mathrm{J} 114$ 833-837.

9. Winterbourn CC, Hampton MB (2008) Thiol chemistry and specificity in redox signaling. Free Radic Biol Med 45: 549-561.

10. Lavoie ME, Rabasa-Lhoret R, Doucet E, Mignault D, Messier L, et al. (2010) Association between physical activity energy expenditure and inflammatory markers in sedentary overweight and obese women. Int J Obes (Lond) 34 1387-1395.

11. Faraj M, Messier L, Bastard JP, Tardif A, Godbout A, et al. (2006) Apolipoprotein $B$ : a predictor of inflammatory status in postmenopausal overweight and obese women. Diabetologia 49: 1637-1646.

12. Schectman G, Patsches M, Sasse EA (1996) Variability in cholestero measurements: comparison of calculated and direct LDL cholesterol determinations. Clin Chem 42: 732-737.

13. Jialal I, Devaraj S, Venugopal SK (2004) C-reactive protein: risk marker or mediator in atherothrombosis? Hypertension 44: 6-11.

14. Matthews DR, Hosker JP, Rudenski AS, Naylor BA, Treacher DF, et al. (1985) Homeostasis model assessment: insulin resistance and beta-cell function from fasting plasma glucose and insulin concentrations in man. Diabetologia 28 412-419.

15. Abdul-Ghani MA, Matsuda M, Balas B, DeFronzo RA (2007) Muscle and liver insulin resistance indexes derived from the oral glucose tolerance test Diabetes Care 30: 89-94.

16. Bastard JP, Vandernotte JM, Faraj M, Karelis AD, Messier L, et al. (2007) Relationship between the hyperinsulinemic-euglycaemic clamp and new simple index assessing insulin sensitivity in overweight and obese postmenopausal women. Diabetes Metab 33: 261-268

17. Karelis AD, Faraj M, Bastard JP, St-Pierre DH, Brochu M, et al. (2005) The metabolically healthy but obese individual presents a favorable inflammation profile. J Clin Endocrinol Metab 90: 4145-4150. 
Citation: Lavoie M, Rabasa-Lhoret R, Ziai S, Lavoie J (2011) Blood Glutathione Peroxidase Activity in Relation with the Risk of Cardiovascular Diseases in Obese Women. J Diabetes Metab 2:136. doi:10.4172/2155-6156.1000136

18. Griffith OW (1980) Determination of glutathione and glutathione disulfide using glutathione reductase and 2-vinylpyridine. Anal Biochem 106: 207-212.

19. Lavoie JC, Chessex P (1997) Gender and maturation affect glutathione status in human neonatal tissues. Free Radic Biol Med 23: 648-657.

20. Chessex P, Lavoie JC, Laborie S, Vallee J (1999) Survival of guinea pig pups in hyperoxia is improved by enhanced nutritional substrate availability for glutathione production. Pediatr Res 46: 305-310.

21. Becker K, Wilkinson AR (1993) Flavin adenine dinucleotide levels in erythrocytes of very low birthweight infants under vitamin supplementation. Biol Neonate 63: 80-85

22. Corti R, Fayad ZA, Fuster V, Worthley SG, Helft G, et al. (2001) Effects of lipid-lowering by simvastatin on human atherosclerotic lesions: a longitudinal study by high-resolution, noninvasive magnetic resonance imaging. Circulation 104: 249-252.

23. Rabasa-Lhoret $R$, Laville $M$ (2001) How to measure insulin sensitivity in clinical practice? Diabetes Metab 27: 201-208.

24. Surapaneni KM, Venkataramana G (2007) Status of lipid peroxidation, glutathione, ascorbic acid, vitamin $\mathrm{E}$ and antioxidant enzymes in patients with osteoarthritis. Indian J Med Sci 61: 9-14.

25. Tinahones FJ, Murri-Pierri M, Garrido-Sanchez L, Garcia-Almeida JM, GarciaSerrano S, et al. (2009) Oxidative stress in severely obese persons is greater in those with insulin resistance. Obesity (Silver Spring) 17: 240-246.

26. Oliveira AC, Parise ER, Catarino RM, Lanzoni V, Leite-Mor MM, et al. (2009) Insulin resistance and not steatosis is associated with modifications in oxidative stress markers in chronic hepatitis C, non-3 genotype. Free Radic Res 43: 1187-1194.

27. Brons C, Jensen CB, Storgaard H, Hiscock NJ, White A, et al. (2009) Impact of short-term high-fat feeding on glucose and insulin metabolism in young healthy men. J Physiol 587: 2387-2397.

28. Kraegen EW, Clark PW, Jenkins AB, Daley EA, Chisholm DJ, et al. (1991) Development of muscle insulin resistance after liver insulin resistance in highfat-fed rats. Diabetes 40: 1397-1403.
29. Wang J, Obici S, Morgan K, Barzilai N, Feng Z, et al. (2001) Overfeeding rapidly induces leptin and insulin resistance. Diabetes 50: 2786-2791.

30. Bonora E, Kiechl S, Willeit J, Oberhollenzer F, Egger G, et al. (2007) Insulin resistance as estimated by homeostasis model assessment predicts inciden symptomatic cardiovascular disease in caucasian subjects from the general population: the Bruneck study. Diabetes Care 30: 318-324.

31. Faraj M, Lavoie ME, Messier L, Bastard JP, Prud'homme D (2010) Reduction in serum apoB is associated with reduced inflammation and insulin resistance in post-menopausal women: A MONET study. Atherosclerosis 211: 682-688.

32. Superko HR, Gadesam RR (2008) Is it LDL particle size or number that correlates with risk for cardiovascular disease? Curr Atheroscler Rep 10: 377 385 .

33. Garcia-Pinilla JM, Galvez J, Cabrera-Bueno F, Jimenez-Navarro M, GomezDoblas JJ, et al. (2008) Baseline glutathione peroxidase activity affects prognosis after acute coronary syndromes. Tex Heart Inst J 35: 262-267.

34. Gill R, Tsung A, Billiar T (2010) Linking oxidative stress to inflammation: Tolllike receptors. Free Radic Biol Med 48: 1121-1132.

35. Zhang X, Min X, Li C, Benjamin IJ, Qian B, et al. (2010) Involvement of reductive stress in the cardiomyopathy in transgenic mice with cardiac-specific overexpression of heat shock protein 27. Hypertension 55: 1412-1417.

36. Matsuzawa-Nagata $\mathrm{N}$, Takamura $\mathrm{T}$, Ando $\mathrm{H}$, Nakamura $\mathrm{S}$, Kurita $\mathrm{S}$, et al. (2008) Increased oxidative stress precedes the onset of high-fat diet-induced insulin resistance and obesity. Metabolism 57: 1071-1077.

37. Ceriello A, Motz E (2004) Is oxidative stress the pathogenic mechanism underlying insulin resistance, diabetes, and cardiovascular disease? The common soil hypothesis revisited. Arterioscler Thromb Vasc Biol 24: 816-823.

38. Lee OJ, Schneider-Stock R, McChesney PA, Kuester D, Roessner A, et al (2005) Hypermethylation and loss of expression of glutathione peroxidase-3 in Barrett's tumorigenesis. Neoplasia 7: 854-861.

39. Peng DF, Razvi M, Chen H, Washington K, Roessner A, et al. (2009) DNA hypermethylation regulates the expression of members of the Muclass glutathione S-transferases and glutathione peroxidases in Barrett's adenocarcinoma. Gut 58: 5-15. 